\title{
VISCERAL LEISHMANIASIS IS ENDEMIC IN GOLDEN JACKALS OF BANGLADESH AGRICULTURAL UNIVERSITY CAMPUS, A THREAT FOR EXPANDING FUTURE ZOONOTIC VISCERAL LEISHMANIASIS
}

\author{
M. A. H. N. A. Khan*, S. S. Khanm, J. Bashu, U. K. Rima, M. Pervin, M. Z. Hossain, M. A. Habib, \\ G. A. Chowdhury and M. M. Hossain \\ Departments of Pathology, Faculty of Veterinary Science, Bangladesh Agricultural University, \\ Mymensingh-2202, Bangladesh.
}

\begin{abstract}
Visceral leishmaniasis (VL), now a day, is an endemic protozoal disease of human in Bangladesh. The disease is transmissible to canids and endemic in canids in many parts of the world including western China and India. In Bangladesh, the occurrence of VL in canids is unknown. Golden jackals, a common wild canid of Bangladesh Agricultural University (BAU) Campus, living with street dogs, cats and often get closure to human shelter. It is not surprising to find VL in golden Jackals of BAU campus, Mymensingh and this study was, therefore, aimed to identify the occurrence of leishmanial infection in their visceral organs. Venous blood, liver, kidney, spleen and bone marrow from the jackals $(\mathrm{N}=5)$ were collected, impression smears from these organs were made onto clean slides and stained with Giemsa's stain. Leishmanial pro - and amastigote stages of the parasite were detected in the impression smears made from liver, spleen, kidney and bone marrow in all jackals. Leishmanial amastigote stage of the parasite was seen in the macrophages of blood, spleen and liver. Part of liver, kidney, spleen and bone marrow from the jackal were fixed in $10 \%$ buffered neutral formalin and stained with hematoxylin and eosin (H\&E). Histopathological study revealed scatteredly distributed microgranuloma in the hepatic parenchyma, consisting of closely packed collection of macrophages and lymphocytes with fibrous tissue encapsulation. In kidney, chronic glomerulonephritis was seen; the inflamed areas were infiltrated with lymphocytes and macrophages. Specific pathology in spleen and bone marrow was not observed. Genomic DNA was extracted from the liver, kidney, spleen and bone marrow of jackals. Polymerase chain reaction (PCR) with species specific primers were used to identify the species of leishmanial protozoa involved. Results of PCR showed 145bp amplicon, specific for Leishmania donovani infection in all five jackals. This is the first report in Bangladesh describing the occurrence of VL in golden jackals. This result reinforce the assumption that golden jackals of BAU campus harbour leishmanial protozoa, could serve as a symptomless carrier and transmit the infection to other canids and human, require further investigation.
\end{abstract}

Key words: Golden jackals, Visceral leishmaniasis, Giemsa's staining, PCR

\section{INTRODUCTION}

The leishmaniasis are a group of diseases transmissible between humans and animals, caused by obligate intracellular protozoa of the genus Leishmania and transmitted by the bite of various species of phlebotomine sand-flies (Bailey and Lockwood, 2007). There are researches suggested that the domestic dog (Canis familiaris), which is already incriminated as the primary reservoir host of zoonotic visceral leishmaniasis (ZVL) and may have a reservoir role in the domestic transmission of human cutenious leishmaniasis caused by Leishmania braziliensis, L. panamensis, and L. peruviana (Reithinger and Davies. 1999). Canine leishmaniasis is a widespread protozoan infection in South Asian countries and caused by Leishmania donovani (L. donovani) complex (Alvar et al., 2006). Dog is the domestic reservoir of the parasite (Bryceson, 1996) but other canids could have served as carrier of infection. Visceral leishmaniasis has been described in red foxes in Italy (Mancianti et al., 1994; Dipineto et al., 2007), France (Rioux et al., 1968), Portugal (Abranches et al., 1983) and Spain (Criado-Fornelio et al., 2000; Dantas-Torres., 2007) and prevalent throughout the tropical and sub-tropical regions of Africa, Asia, the Mediterranean, Southern Europe (old world) and South and Central America (new world). The disease in human is endemic in 61 countries and is responsible for the annual disability of 1.81 million people and 57,000 lives (Gonzalez et al., 2009; Reithinger and Davies., 2002). Greater than $90 \%$ of VL cases occur in Bangladesh, Ethiopia, Brazil, India, Nepal and Sudan (WHO, 2005), while the equivalent number of cases of cutaneous leishmaniasis occur in Afghanistan, Algeria, Brazil, Iran, Peru, Saudi Arabia and Sudan (Khanjani et al., 2009).

*Corresponding e-mail address: hadikhan68@yahoo.co.uk

Copyright (C 2012 Bangladesh Society for Veterinary Medicine

All rights reserved 0253/2012 


\section{A. H. N. A. Khan and others}

Clinically and epidemiologically, there are two main forms of VL in canids: zoonotic visceral leishmaniasis (ZVL) and anthroponotic visceral leishmaniasis (Reithinger and Davies. 1999). ZVL is caused by L. donovani (Srivastava et al., 2011), L. infantum or L. chagasi and is distributed in Europe, Asia, Africa, and Latin America (Lise et al., 2010). In most of these regions canine infection with L. infantum is the cause and therefore, act as reservoir of infection for human VL. Accurate and rapid detection of VL in canids is difficult due to its variable symptomology and as well as lacking of proper diagnostic facilities. The prevalence of canine leishmanial infection in endemic areas is very important for the epidemiological investigation, zoonotic point of view and as well as adopt future control strategies.

Parasitological, serological and PCR detection were extensively used to investigate canine clinical or subclinical infection with Leishmania organism (Quaresma et al., 2009 ; Admitriy et al., 2011; Veronica 2011). The prevalence of VL in the canids has never been investigated in Bangladesh. This study was aimed to investigate the occurrence of leishmanial infection in golden jackal of BAU campus. Impression smears from the liver, spleen, bone marrow and blood was stained with Giemsa's to identify the presence of either the pro or amastigote stages of the parasites. A systemic gross and microscopic investigation on to the visceral organs of jackals were also carried to evaluate host response following subclinical infection. Studies demonstrated that PCR method is more sensitive and specific than parasitological and serological methods (Criado-Fornelio et al., 2000; Bogdan et al., 2001; Leontides et al., 2002: Cortes et al., 2004 ). Therefore, three sets of primers in PCR amplification were used to amplify genomic DNA of L. donovani, L. brasiliensis and L. infantum (Table 1) from the internal organs to incriminate golden jackal as carrier of VL. The diagnosis of VL in jackal was made principally based on the results of characteristics impression smears staining, gross and microscopic lesions in visceral organs and results of PCR analysis.

\section{MATERIALS AND METHODS}

Randomly selected and sexually mature five golden jackals of both sexes were collected from the residential area of BAU campus, Mymensingh during the period from June 2010 to December, 2011. Systemic dissection and investigation were carried out onto visceral organs. Venous blood, liver, spleen, kidney and bone marrow were examined using Giemsa's staining, hematoxylin and eosin (H\&E) staining and PCR amplification for the detection and differentiation of the species of protozoa involved in the disease processes.

\section{Examination of impression smears}

A thin smear of venous blood, smears from the liver, spleen, kidney and bone marrow of golden jackals were made onto clean, grease free slide, dried in air and fixed in absolute ice cold methanol (acetone free) for $15 \mathrm{~min}$. The slides were dried in air and stained for 40min in working Giemsa's solution (Luna, 1968). Slides were washed in running tape water for 30sec, dried in air and examined at low (10x) and high power (40x and 100x) microscopic field. An extensive investigation for the presence of pro - and amastigote stages of leishmanial protozoa in blood, in tissue spaces and in macrophages of various organs were employed and protozoal morphology was investigated and images were captured.

\section{Histopathological investigation of tissue changes}

During necropsy spleen, liver, kidney and bone marrow were collected and fixed in 10\% buffered neutral formalin. Formalin fixed tissue samples were trimmed, processed, sectioned and stained with H\&E (Luna, 1968). A low $(10 x)$ and high power $(40 x, 100 x)$ microscopic analysis were carried out to observe the changes in the internal organs specific to VL. Emphasis was given to investigate the presence of amastigote stage of leishmanial protozoa in macrophages.

\section{PCR detection of the species of Leishmania}

Genomic DNA of golden jackals was extracted from the liver, spleen, kidney and bone marrow as per manufacturer's instructions (Promega Corporation, Madison, USA). The DNA samples were evaluated quantitatively and qualitatively using spectrophotometry $\left(\mathrm{A}^{260}\right.$ and $\left.\mathrm{A}^{280}\right)$ and agarose gel electrophoresis. Three sets of primers (Table 1) were used to identify the species of leishmanial protozoa involved in the disease processes. PCR reactions were performed onto each DNA sample in $20 \mu 1$ reaction volume containing nuclease free water, 2x PCR master mix (Promega Corporation, USA), 20pmol forward and 20pmol reverse primers and $300 \mathrm{ng}$ to $350 \mathrm{ng}$ DNA template. A total of 45 cycles of DNA amplification reaction for L. donovani and $L$. 
brasiliensis and 30 cycles for L. infantum were carried out. The thermal profile used for L. donovani comprised an initial denaturation for two minutes at $94^{\circ} \mathrm{C}$ followed by 45 cycles of DNA amplification reaction in a Master Cycler (Master Cycler Gradient, Eppendorf, Germany). The condition of PCR amplifications were denaturation for $60 \mathrm{sec}$ at $94^{\circ} \mathrm{C}$, primer annealing for $90 \mathrm{sec}$ at $62^{\circ} \mathrm{C}$ and extension for $30 \mathrm{sec}$ at $70^{\circ} \mathrm{C}$ followed by a final extension for $10 \mathrm{~min}$ at $70^{\circ} \mathrm{C}$. The thermal profile used for L. brasileiensis comprised an initial denaturation for $4 \mathrm{~min}$ at $94^{\circ} \mathrm{C}$. The conditions of amplifications in PCR System were denaturation for $30 \mathrm{sec}$ at $94^{\circ} \mathrm{C}$, primer annealing for $30 \mathrm{sec}$ at $50^{\circ} \mathrm{C}$ and extension for $30 \mathrm{sec}$ at $72^{\circ} \mathrm{C}$ followed by a final extension for $10 \mathrm{~min}$ at $70^{\circ} \mathrm{C}$. The thermal profile used for L. infantum comprised an initial denaturation for $4 \mathrm{~min}$ at $94^{\circ} \mathrm{C}$. The conditions of amplifications in PCR System were denaturation for $45 \mathrm{sec}$ at $94^{\circ} \mathrm{C}$, primer annealing for $45 \mathrm{sec}$ at $50^{\circ} \mathrm{C}$ and extension for $30 \mathrm{sec}$ at $72^{\circ} \mathrm{C}$ followed by a final extension for $10 \mathrm{~min}$ at $70^{\circ} \mathrm{C}$. The PCR reactions were finally terminated by adding $3 \mu \mathrm{l} 50 \mathrm{mM}$ EDTA and PCR products were analyzed by electrophoresed in $2 \%$ agarose gel, stained with ethidium bromide and examined under UV light using an image documentation system (Cell Biosciences, Alphalmager HP, USA).

Table 1. Primers and their sequences used to identify the species of leishmanial organism in golden jackals at BAU campus

\begin{tabular}{|l|l|c|c|l|}
\hline Primers name & Sequence & $\begin{array}{c}\text { Product } \\
\text { Size }\end{array}$ & $\begin{array}{c}\text { Species of } \\
\text { organism }\end{array}$ & References \\
\cline { 1 - 2 } LDF & 5'-CTTTTCTGGTCCCGCGGGTAGG-3' & \multirow{2}{*}{$145 \mathrm{bp}$} & L. donovani & Gomes et al., 2007 \\
\cline { 1 - 2 } LDR & 5'-CCACCTGGCCTATTTTACACCA-3' & \multirow{2}{*}{$120 \mathrm{bp}$} & L. brasiliensis & Passos et al., 1999 \\
\cline { 1 - 2 } LBF & 5'-GGCCCACTATATTACACCAACCC-3' & \multirow{2}{*}{$130 \mathrm{bp}$} & L. infantum & Lachaud et al., 2002 \\
\cline { 1 - 2 } & 5'-GGGGTAGGGGCGTTCTGCGAA-3' & 5'AATCGAGCAGCTCAAGGAAG-3' & &
\end{tabular}

\section{RESULTS AND DISCUSSION}

A systemic necropsy, impression smear staining with the Giemsa's, detailed histopathology and species specific PCR were applied to know the occurance of VL in golden jackals of BAU campus. The PCR amplification protocol appeared most sensitive tool in terms of detecting specific infections with L. donovani, L. brasiliensis and L. infantum in golden jackals. As the animals were subjected to thorough necropsy, genomic DNA was extracted from the internal organs to confirm the presence of leishmanial species in the visceral organs where most lesions were seen.

In this study, the golden jackals investigated were apparently healthy and major gross changes characteristics of VL were not observed either in spleen, liver, kidney and bone marrow. However, in common the spleen appeared relatively larger. Results of impression smears staining showed the presence of leishmanial amastigotes in circulating monocytes (while the jackals were collected at night) and macrophages of spleen (Figure 1). Promastigote stages of the parasite was commonly detected in the impression smears prepared from spleen, liver, kidney and bone marrow (Figure 2). The concentration of leishmanial promastigotes (per 10x microscopic field) appeared higher in the impression smears prepared from spleen $(\mathrm{N}=10-23)$, followed by kidney $(\mathrm{N}=5-7)$, liver $(\mathrm{N}=4-6)$ and bone marrow $(\mathrm{N}=3-4)$. The promastigotes (Figure 2) possesses a long tail and a head with variable morphology. Under oil immersion microscopy the head showed variable morphology like sperm head shaped, club shaped, heart shaped, oval shaped or sometimes in balloon shaped (Figure 2). A tail of variable length (about $5-70 \mu \mathrm{m})$ and width $(0.2-1 \mu \mathrm{m})$ was found to attach with the body of the protozoa $(7-12 \mu \mathrm{m})$. Using smear microscopy, the species of leishmania involved whether pathogenic or non pathogenic remain undetected. During impression smear staining the morphological pattern of the head and tail of the protozoa revealed that while the protozoa turn in pre-promastigote stage (Figure 1, b) released in blood, possesses very tiny tail, later on the tail grow bigger, swim in blood before infecting/ engulfing by new macrophages. About 5-15 pre-promastigotes were found to liberate from each infected macrophage (Figure 1, a \& b). The reason of not detecting promastigote phases of the protozoa in the circulating blood of jackals could be due to nocturnal affinity of the 


\section{A. H. N. A. Khan and others}

protozoa (Torres, 2007) and the animals collected and examined at day time. However, comparative data on to the morphologic analysis of the developmental stages of lerishmanial protozoa in man and animals is very limited. Al-Shanawi (1975) investigated clinically suspected cases of visceral leishmaniasis $(83.4 \%)$ in canids and identified positive cases by examining bone marrow. However studies showed that bone marrow examination was the least sensitive method for the detection of visceral leishmaniasis (Zijlstre et al., 1992; Jassim, 1998; Ataya et al., 2001). Our study showed that impression smears prepared from sternum and stained with Giemsa's showed promastigotes, however, the number per 10x microscopic field were relatively lower compared to the load of promastigotes in spleen, kidney and liver. The load of promastigotes per microscopic field in the impression smears of spleen were always appeared higher compared to other organs.

Results of histopathological examination showed scatterdly distributed nodular lesion in liver (Figure 3a), consisting of closely packed collection of lymphocytes, macrophages and circumscribed by fibrous connective tissue. Most striking lesion seen was around the central veins (Figure 3b) of liver and there was lysis of affected hepatocytes. The necrotic area was desolated and few migratory stages of leishmanial protozoa was seen as elongated body. The leishmanial stages could have entered into the veins and assumed to infect remote tissue. In kidney, extensive glomerulonephritis was seen. There was profuse infiltration of lymphocytes, eosinophils and macrophages in the affected glomeruli and glomerular capsules were distorted. Renal tubules found to contain light pink to purple color precipitate in their lumen and in some instances the tubules were blocked with proteinaceous renal cast. Glycogen deposition was also seen in renal tubules and this change may or may not relate to leishmanial infection. Notable changes in the bonemerrow was not seen in this study. Lujan et al. (1986) described granulomatous leishmanial lesions in monkey, consisted of macrophages containing parasites, lymphocytes, plasma cells, and occasionally eosinophils. Differences were not observed between promastigote or amastigote derived nodular lesion or strains of leishmanial protozoa involved, course of infection and variation in either the sex and ages of the hosts and geography.

In general the clinical signs due to VL in canids were variable and asymptomatic infections mostly occured in canids. In symptomatic cases, common visible signs reported were lethargy, weight loss, a decreased appetite, anemia, splenomegaly and local or generalized lymphadenopathy. Fever can be intermittent, and is absent in many cases (Calabri et al., 1997). Chronic renal disease is common in foxes infected with L. infantum; it may be the only syndrome, and it is often the cause of death (Criado-Fornelio et al., 2000). Some animals may also developed ocular, skin or mucosal lesions, sneezing, chronic diarrhea, vomiting, chronic relapsing colitis, chronic hepatitis, osteolytic or osteoproliferative leishmaniasis (Mancianti et al., 1986). In this study, the jackals investigated appeared healthy at necropsy and gross lesion was not seen. Microscopically there was granulomatous reaction in the liver parenchyma. The nodule consisting of closely packed collection of macrophages replacing hepatic parenchyma (Figure 3a) but lacking of caseous necrotic centre. The reason for granulomata formation in liver following VL infection could be due to parasitized macrophages tended to accumulate, invite more reactive cells leading necrosis in the inflamed tissue and proliferation of connective tissues around tissue reaction (Tryphonas et al., 1977). The hepatic parenchyma around the central vein was degenerated, necrotic and dissolute. There were numerous darkly stained promastigotes distributed widely around the necrosed area (Figure $3 b$ ). The promastigote stages of the protozoa were found inside the congested central vein indicating stage of dissemination via blood. However, most notable lesion as seen in golden jackal was chronic nephrotic syndrone and renal failure could have linked with VL in canids.

The lifecycle of Leishmania indicated a free living stages of the protozoa (promastigote) in the gut of the sand fly (Phlebotomus). However, free living stages of the protozoa in the blood of the mammals can be seen immediately after the bite of sand flies and before engulfing by the macrophages (Matthew et al., 1963). In this study results of the impression smears staining revealed free living stages (Figure 2) in the spleen, liver, kidney and bone marrow. It may be concluded that the free living stage (promastigote) of Leishmania is cyclically developed in mammals from amastigote stage in the macrophages. The free living stages reside in the visceral organs before they infect or disseminate nearby by tissue or remote tissue through circulation. However, using impression smears staining amsatigote and promastigote stages of the parasite was easily detected but in order to detect species of the protozoa involved require molecular technique especially PCR

PCR assays have greatly improved the sensitivity and specificity to diagnose Leishmanial infection in canids (Torres et al., 2007). However, to make this technique achievable, the sampling methods need to be noninvasive, easy and painless. Conjunctival swab (CS) presented all these characteristics, together with high sensitivity. 
Using PCR on to the DNA from CS diagnosed symptomatic dogs successfully and observed $92 \%$ sensitivity (Strauss et al. 2004). However, Ferreira et al. (2008) found 91.7\% and Pilatti et al. (2009) obtained between 73.9 and $95.6 \%$ sensitivity depending upon the PCR method used to diagnose VL. In this study as the animals were examined at necropsy, PCR amplification (Table 1) with the DNA template from the liver, spleen, kidney and bonemarrow of golden jackal were carried out (Figure 4) using primers specific for L. donovani(4a, lane 1-5), $L$. brasiliensis (4b, lane 6-10) and L. infantum (4c, lane 11-15). But It was not possible to determine the sensitivity and specificity of the PCR we used due to lack of samples from animals with known disease status. PCR amplification with the DNA from these organs showed 145bp amplicon indicating infectivity of all jackals with L. donovani. PCR amplification using primers specific for L. brasiliensis showed the presence of $120 \mathrm{bp}$ amplicon in one case and could have infected with $L$. brasiliensis. However, PCR amplification of DNA template using primers specific to $L$. infantum did not develop any amplicon in any of the jackals. This study provide evidence that the jackal $(\mathrm{N}=5)$ at BAU campus were infected with $L$. donovani and one jackal was co-infected with $L$. donovani and L. brasiliensis. Infectivity of jackals at BAU campus with L. infantum was not detected in this study. Dipineto et al. (2007) also used PCR assay and recorded 40\% (20/50) infectivity in foxes with Leishmania sp. Whereas Bettini et al. (1980) and Mancianti et al. (1994) used indirect immunofluorescence assay, enzyme-linked immunosorbent assay, and microscopy for detection of Leishmania , and reported $18 \%$ $(9 / 50)$ and $6 \%(1 / 16)$ infectivity of foxes respectively. As there was lacking data regarding infectivity of jackals with Leishmania, to the best of our knowledge this is the first report describing the occurrence of leishmanial infection (VL) in jackals at BAU campus in Bangladesh. The result may come to an assumption that jackals can be considered as an important reservoir for leishmanial infection similar to other wild canids (Michalsky et al., 2007).

\section{Threat for zoonotic visceral leishmaniasis (ZVL)}

The emergence of ZVL as an increasing public health problem is mainly due to demographic and ecological factors. ZVL now occurs in many regions of Latin America where it was not previously reported. In South America, especially in Brazil, Colombia and Venezuela, migration and urbanisation have largely contributed to the increasing public health problem posed by ZVL. A prime example is the massive migration that was occurred in north-eastern Brazil where, due to a long-lasting drought and its socio-economic consequences (ruined crops, starvation and miserable conditions), people had to abandon their rural location to settle in the hastily constructed shanty towns at the periphery of large cities such as Petrolina, Fortaleza, Sobral, Natal, Joao Pessoa, Sao Luis, Teresina, Jacobina and, more recently Salvador do Bahia (Jeronimo et al., 1994; Arias et al., 1996). In poor suburbs, Lu. longipalpis, the principal Neotropical vector of L. infantum, is everywhere, dogs are numerous, sanitary conditions are poor and malnutrition is frequent. The new migrants often bring dogs, chickens and pigs with them and keep these animals in and around their houses. These conditions create an excellent habitat for vector Lu. longipalpis (Tesh, 1995; Desjeux, 2002). Thus most of the canids in general, fulfill the required attributes to be efficient reservoirs of leishmanial infection (Torres and Filho, 2006). Due to its close relationship with humans, the domestic dog has long been implicated as the main reservoir of L. infantum in China, the Mediterranean basin and the Americas (Lainson and Rangel, 2005; Torres and Filho, 2006). Similar situation could have existed in Bangladesh, where cattle, sheep, goat, dogs and domestic cats are found to live in a habitat and allowing free living vector sandflies to bite and suck blood. Jackal is a common canid in Bangladesh and now a day are found to eat with dogs and cats in a same tale at BAU campus. Leishmanial infection is common in jackals at BAU campus. It is not surprising to find leishmanial infection in dogs and cats living in the teritorry (study is in progress) of BAU. It is plausible to conclude that the semidomestic jackals at BAU campus with the domestic dogs could play role in the zoonotic transmission of visceral leishmaniasis. It is virtually not possible to eliminate all the vectors of VL and carrier jackals from BAU campus and its surroundings. However, it needs to know whether human being living at BAU campus also infected with Leishmania, thus early attempt can be taken to prevent the future casualty. 

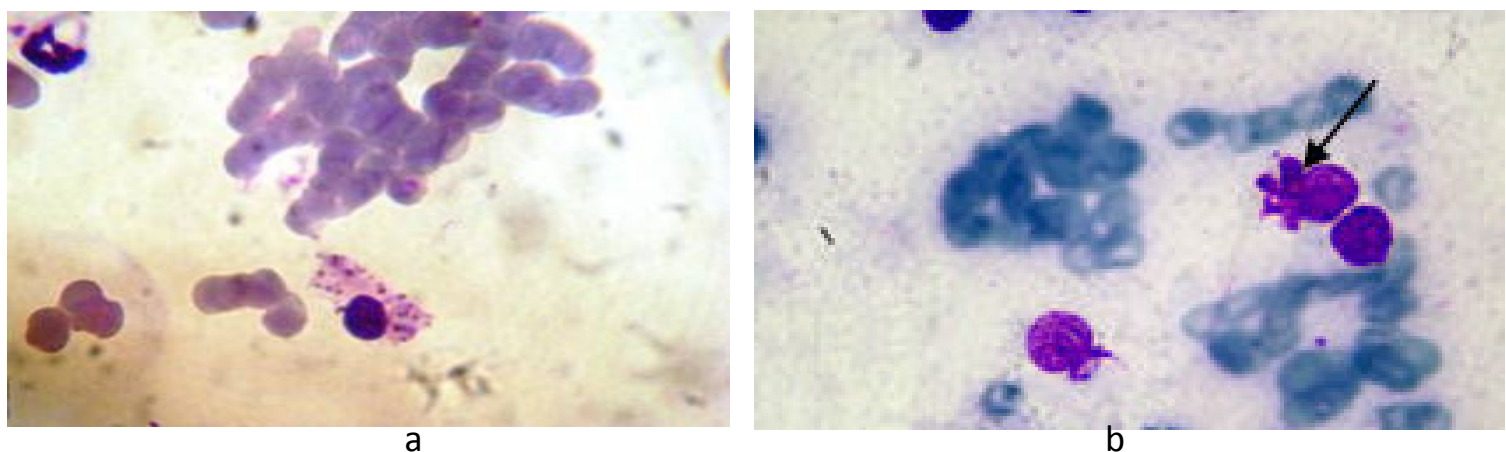

Figure 1. Impression smears prepared from peripheral blood (a) and spleen (b) of a golden jackal and stained with Giemsa's (100x). Dark blue colour amastigotes (red arrow, left) inside the monocyte of blood was seen. A stage between pro and amastigote was seen inside the macrophages of spleen (black arrow, right) and the protozoa found to throw its tiny tail through cell wall before releasing into extracellular stage (b).

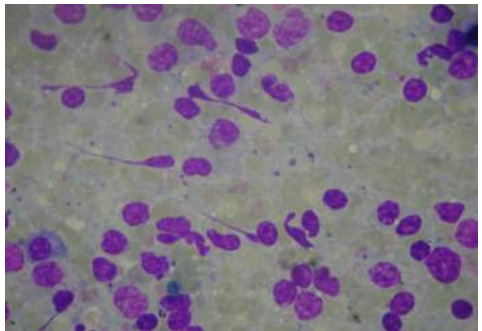

$\mathrm{a}(40 \mathrm{x})$

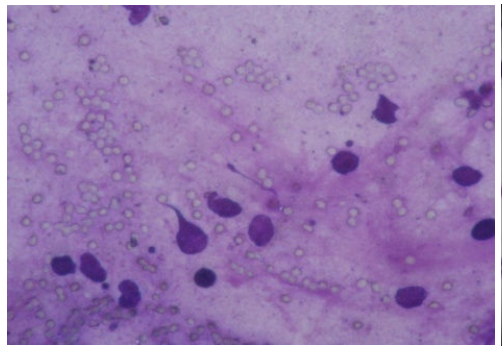

$\mathrm{d}(40 \mathrm{x})$

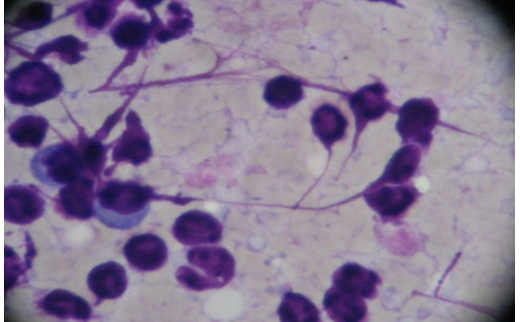

b (100x)

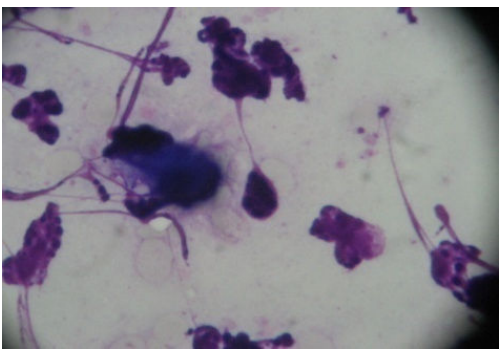

e $(100 x)$

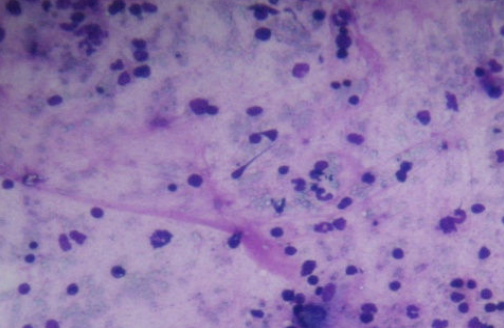

$\mathrm{c}(10 \mathrm{x})$

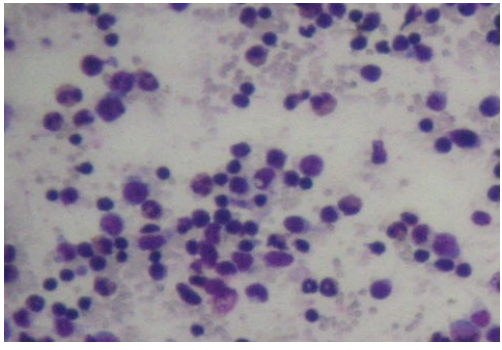

$f(10 x)$

Figure 2. Morphological investigation of promastigote stages of leishmanial protozoa (black arrow) onto the organ impression smears prepared from golden jackals and stained with Giemsa's. The promastigote stage of the parasite was seen in the intercellular spaces and found to contain a tail (white arrow) and a head (black arrow) with variable morphology. Under oil immersion microscopy sperm head shaped (a, black arrow), balloon shaped (b and e, black arrow) and sometimes tadpole shaped (d, black arrow) appearance of the head of the protozoa were seen compared to non-infected control (f, red arrow) lacks promastigote. 


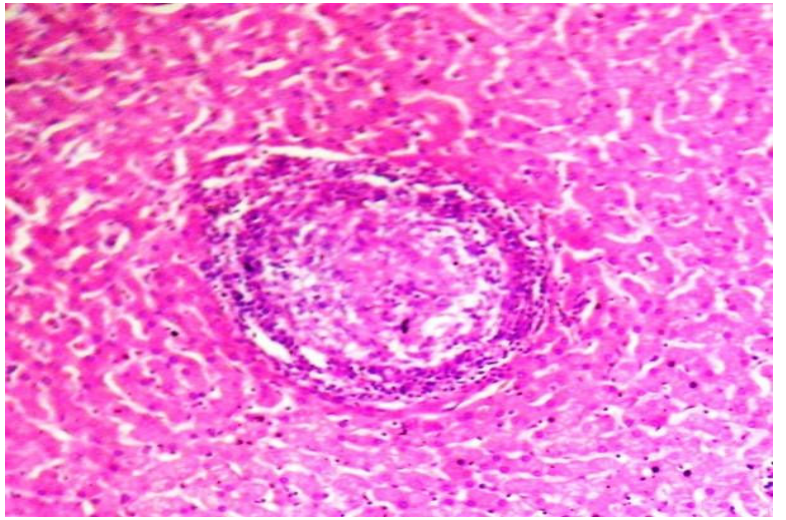

a

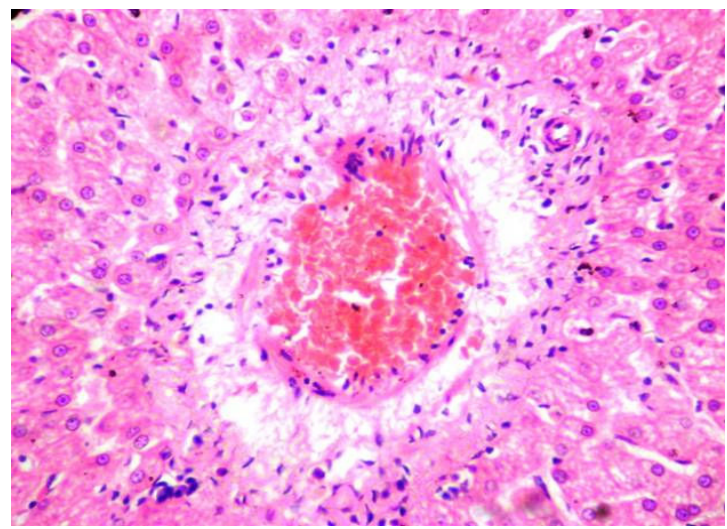

b

Figure 3. Section of the liver of a golden jackal ( $a$ and $b$ ) and stained with H\&E. Scatteredly distributed granulomatous nodule (black arrow) was seen in the liver parenchyma (left). The nodule consisting of closely packed collection of macrophagess replacing hepatic parenchyma and lacking caseous necrotic centre $(40 \mathrm{x})$. There was dissolution of hepatic parenchyma around the central vein (b). Numerous darkly stained rod shaped promastigotes (red arrow) in the necrotic zone around and close to the central vein were seen. Few of the leishmanial promastigotes (green arrow) were seen entering the congested central vein indicating stage of dissemination of the protozoa $(100 \mathrm{x})$.

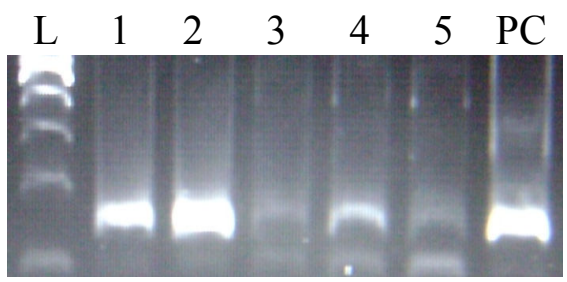

a

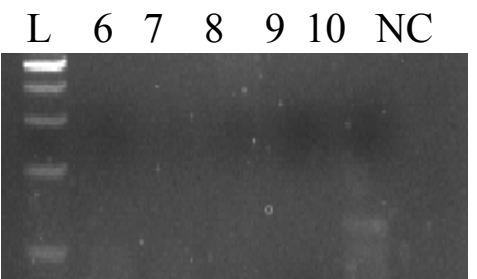

b

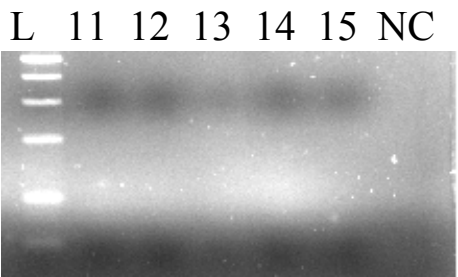

Figure 4. PCR amplification of DNA template obtained from the liver of golden jackals $(\mathrm{n}=5)$ and amplified using primers specific for L. donovani (a, lane 1-5), L. brasiliensis (b, lane 6-10) and L.infantum (c, lane 11-15). Lane L are for $100 \mathrm{bp}$ DNA ladder, lane PC known positive control for $L$. donovani and lane NC are for negative control. PCR amplification showed the presence of $L$. donovani (145bp, a) in jackals 1, 2, 3, 4 and 5. PCR amplification using primers specific for L. brasiliensis showed the presence of L. brasiliensis (120bp, b) in a jackal (jackal No. 5, lane 10). PCR amplification of DNA template using primers specific for $L$. infantum did not develop any amplicon in any of the jackals (c, lane 11-15).

\section{REFERENCES}

1. Al-Shanawi F (1975). Comparison of the course of laboratory infection in Iraqi strain of Leishmania donovani. MSc Thesis, College of Science, University of Baghdad.

2. Abranches P, Conceicao-Silva FM, Silva And and Pereira MCD (1984). Kala-azar in Portugal. The sylvatic cycle in the enzootic focus of Arrabida. Journal of Tropical Medicine and Hygiene 87: $197-200$.

3. Admitriy K, Ashavkat R, Nevgeny P, Alon W, Mrokhat N, Ivalentina P, Aaziza F, Abdelmajeed N, Eyal K, Zmohammad A, Flionel S, Lchrales C, Gabriele S and Gad B (2011). Canine leishmaniosis and its relationship to human visceral leishmaniasis in Eastern Uzbekistan. Parasite Vectors 4: 58. 28.

4. Alvar J, Yactayo S and Bern C (2006). Leishmaniasis and poverty. Trends in Parasitology 22:552-557.

5. Arias JR, Monteiro PS and Zicker F (1996). The re-emergence of visceral leishmaniasis in Brazil. Emerging Infectious Diseases 2:145-146. 


\section{A. H. N. A. Khan and others}

6. Ataya A and Sarji NA (2001). Comparative study of methods used for the diagnosis of leishmaniasis in Syria. Journal of the Arab Board of Medicine 3: 103-8.

7. Bailey MS and Lockwood DN (2007). Cutaneous leishmaniasis. Clinical Dermatology 25(2): 203-211.

8. Bettini S, Pozio E and Gradoni L (1980). Leishmaniasis in Tuscany (Italy): (II) Leishmania from wild Bodentia and Carnivora in a human and canine leishmaniasis focus. Transactions of the Royal Society of Tropical Medicine and Hygiene 74: 77-83.

9. Bogdan C, Schonian G, Banuls AL, Hide M, Pratlong F, Lorenz E, Rollinghoff M and Mertens R (2001). Visceral leishmaniasis in a German child who had never entered a known endemic area: case report and review of the literature. Clinical Infectious Disease 32 (2): 302-306.

10. Bryceson ADM (1996). Leishmaniasis. In: Tropical Diseases, 12th edn. WB Saunders; London 1213 1245.

11. Calabri GB, Casini T, Cristiano R, Pasquini E, La Cauza F, Lippi A, Grifi G, Cocchi P, Calabri G (1997). Visceral leishmaniasis in children in the province of Florence. Medical and surgical pediatrics 19(6): 441.

12. Cortes S, Rolão N, Ramada J and Campino L (2004). PCR as a rapid and sensitive tool in the diagnosis of human and canine leishmaniasis using Leishmania donovani sl-specific kinetoplastid primers. Transactions of the Royal Society of Tropical Medicine and Hygiene 98: 12-17.

13. Criado-Fornelio A, Gutierrez-Garcia L, Rodriguez-Caabeiro F, Reus-Garcia E, Roldan-Soriano MA and Diaz-Sanchez MA (2000). A parasitological survey of wild red foxes (Vulpes vulpes) from the province of Guadalajara, Spain. Veterinary Parasitology 92: 245-251.

14. Desjeux P (2002). Urbanisation of the leishmaniases. Communicable Disease Surveillance and Response, Emerging Public Health Risks (CSR/EPH), World Health Organization, Geneva, Switzerland page 49-56 (In; International symposium on Canine Leishmaniasis: moving towards a solution. Proceedings of the Second International Canine Leishmaniasis Forum. Held in Sevilla, Spain, 23rd May - 2002.

15. Dipineto L, Manna L, Baiano A, Gala M, Fioretti A, Gravino AE and Menna LF (2007). Presence of Leishmania infantum in red foxes (Vulpes vulpes) in southern Italy. Journal of Wildlife Diseases 43(3): 518-520.

16. Ferreira SA, Ituassu LT, de Melo MN and de Andrade ASR (2008). Evaluation of the conjunctival swab for canine visceral leishmaniasis diagnosis by PCR-hybridization in Minas Gerais State, Brazil. Veterinary Parasitology 152(3-4): 257-263.

17. Gomes AH, Ferreira IM, Lima ML, Cunha EA, Garcia AS, Araujo MF and Pereira-Chioccola VL (2007). PCR identification of Leishmania in diagnosis and control of canine leishmaniasis. Veterinary Parasitology 144: 234-241.

18. Gonzalez U, Pinart M, Rengifo-Pardom M, Macayam A, Alvarm J and Tweed JA (2009). Interventions for American cutaneous and mucocutaneous leishmaniasis (Review). The Cochrane Collaboration 2: 1-42.

19. Jassim AH (1998). The evaluation and application of locally prepared direct agglutination test for the diagnosis and sero-epidemiology of visceral leishmaniasis in Basrah. PhD Thesis, College of Medicine, University of Mustansiryia, Iraq.

20. Jeronimo SMB, Olivera RM, Mackay S, Costa RM, Sweet J, Nascimento ET, Luz KG, Fernandes MZ, Jernigan J and Pearson RD (1994). An outbreak of visceral leishmaniasis in Natal, Brazil. Transactions of the Royal Society of Tropical Medicine and Hygiene 88: 386-388.

21. Khanjani N, Gonzalez U, Leonardi-Bee J, Mohebali M, Saffari M and Khamesipour A (2009). Vaccines for preventing cutaneous leishmaniasis (protocol). The Cochrane Collaboration 2: 1-12.

22. Lachaud L, Marchergui-Hammami S, Chabbert E, Dereure J, Dedet JP and Bastien P (2002). Comparison of Six PCR Methods Using Peripheral Blood for Detection of Canine Visceral Leishmaniasis. Journal of Clinical Microbiology 40(1): 210-215.

23. Lainson R and Rangel EF (2005). Lutzomyia longipalpis and the ecoepidemiology of American visceral leishmaniasis, with particular reference to Brazil: a review. Memórias do Instituto Oswaldo Cruz 100: 811827.

24. Leontides LS, Saridomichelakis MN, Billinis C, Kontos V, Koutinas AF, Galatos AD and Mylonakis ME (2002). A cross-sectional study of Leishmania spp. infection in clinically healthy dogs with polymerase chain reaction and serology in Greece. Veterinary Parasitology 109:19-27. 
25. Lise C, Annelise T, Anne M, Gilles B, Paul R and Dedet JP (2010). Environmental risk mapping of canine leishmaniasis in France. Parasites and Vectors 3: 31.

26. Lujan R, Chapman WL, Hanson WL, Dennis VA (1986). Leishmania braziliensis: Development of primary and satellite lesions in the experimentally infected owl monkey, Aotus trivirgatus. Experimental Parasitology 61(3): 348-358.

27. Mancianti F, Grandoni L, Gramiccia M, Pieri S and Marconcini A (1986). Canine leishmaniasis in the Isle of Elba, Italy. Tropical Medicine and Parasitology 37: 110-2.

28. Mancianti F, Mignone W and Galestri F (1994). Serologic survey for leishmaniasis in free-living red foxes (Vulpes vulpes) in Italy. Journal of Wildlife Diseases 30: 454-456.

29. Matthew E, Rogers PEC, Southgate B A and Harvey AEC (1963). Development of kala-azar in man after inoculation with Leishmania from Kenya sandfly. British Medical Journal 1: 1208-1210.

30. Michalsky É M, Rocha MF, da Rocha Lima A C V M, França-Silva JC, Pires MQ, Oliveira FS, Pacheco RS, dos Santos SL, Barata RA, Romanha ÁJ, Fortes-Dias CL and Dias ES (2007). Infectivity of seropositive dogs, showing different clinical forms of leishmaniasis, to Lutzomyia longipalpis phlebotomine sand flies. Veterinary Parasitology 147(1-2): 67-76.

31. Pilatti MM, Ferreira SA, de Melo M N and de Andrade ASR (2009). Comparison of PCR methods for diagnosis of canine visceral leishmaniasis in conjunctival swab samples. Research in Veterinary Science 87(2): 255-257.

32. Quaresma PF, Murta SM, Ferreira Ede C, da Rocha-Lima AC, Xavier AA and Gontijo CM (2009). molecular diagnosis of canine visceral leishmaniasis: identification of Leishmania species by PCR-RFLP and quantification of parasite DNA by real-time PCR. Acta Tropica 111: 289-294.

33. Reithinger R and Davies CR (1999). Is the domestic dog (Canis familiaris) a reservoir host of american cutenious leishmaniasis? a critical review of the current evidence. American Journal of Tropical Medicine and Hygiene 61(4) : 530-541.

34. Reithinger R and Davies CR (2002). Canine leishmaniasis: novel strategies for control. Trends in Parasitology 18: 289-290.

35. Rioux JA, Albaret JL, Houin R, Dedet JP and AND Lanotte G (1968). Ecologie des leishmanioses dans le sud de la France. 2. Les reservoirs selvatiques. Infestation spontanee de renard (Vulpes vulpes L.). Annales de Parasitologie Humaine et Comparee 43: 421-428.

36. Srivastava P, Mehrotra S, Tiwary P, Chakravarty J and Sundar S (2011). Diagnosis of Indian Visceral Leishmaniasis by Nucleic Acid Detection Using PCR. PLOS Neglected tropical Disease 6(4): e19304.

37. Strauss AD, Charles LJ, Ofer B, Liat G and Gad B (2004). Polymerase chain reaction using noninvasively obtained samples, for the detection of Leishmania infantum DNA in dogs. The Journal of Infectious Diseases 189(9): 1729-1733.

38. Tesh RB (1995). Control of zoonotic visceral leishmaniasis: is it time to change strategies? American Journal of Tropical Medicine and Hygiene 52: 287-292.

39. Torres DF (2007). The role of dogs as reservoirs of Leishmania parasites, with emphasis on Leishmania infantum and Leishmania (Viannia) braziliensis. Veterinary Parasitology 149: 139-146.

40. Torres, DF and Filho BSP (2006). Visceral leishmaniasis in Brazil: revisiting paradigms of epidemiology and control. Revista do Instituto de Medicina Tropical de São Paulo 48: 151-156.

41. Tryphonas L. Zawidzka Z, Bernard MA and Janzen EA (1977). Visceral Leishmaniasis in a Dog: Clinical, Hematological and Pathological Observations. Canine Journal of Comparative Medicine 41(1): 1-12.

42. Veronica M, Javier Q, Armand S, Xavier R, Olga F and Laura A (2011). Canine leishmaniasis: the key points for qPCR result interpretation. Parasites and Vectors 4: 57.

43. Zijlstre EE, Ali MS, El-Hassan AM, El-Toum IA, Satti M (1992). Kala-azar: a comparative study of parasitological methods and the direct agglutination test in diagnosis. Transactions of the Royal Society of Tropical Medicine and Hygiene 86: 505-7.

44. WHO (World Health Organization). (2005). Magnitude of the problem. Available: http://www. who.int/ leishmaniasis/burden/magnitude/burden_magnitude/en/index.html. Accessed 31 May 2013.

45. Zijlstre EE, Ali MS, El-Hassan AM, El-Toum IA and Satti M (1992). Kala-azar: a comparative study of parasitological methods and the direct agglutination test in diagnosis. Transaction of the Royal Society of Tropical Medicine and Hygiene 86: 505-7. 